\title{
1 Enzymatic synthesis of cellulose in space: gravity 2 is a crucial factor for building cellulose II gel 3 structure
}

4 Tomohiro Kuga ${ }^{1}$, Naoki Sunagawa ${ }^{1}$ and Kiyohiko Igarashi ${ }^{1,2^{*}}$

$5{ }^{1}$ Department of Biomaterial Sciences, Graduate School of Agricultural and Life

6 Sciences, The University of Tokyo, 1-1-1 Yayoi, Bunkyo-ku, Tokyo 113-8657,

7 Japan.

$8 \quad{ }^{2}$ VTT Technical Research Centre of Finland, PO Box 1000, Tietotie 2, Espoo FI902044 VTT, Finland.

10 *Corresponding author: aquarius@mail.ecc.u-tokyo.ac.jp

11 ORCID Tomohiro Kuga: https://orcid.org/0000-0001-6120-3344; Naoki Sunagawa: 12 https://orcid.org/0000-0002-0382-6671; Kiyohiko Igarashi: https://orcid.org/0000-0001-5152137177

15 Abstract

16 We previously reported in vitro synthesis of highly ordered crystalline cellulose II by reverse 17 reaction of cellodextrin phosphorylase from the cellulolytic bacterium Clostridium 18 (Hungateiclostridium) thermocellum (CtCDP), but the formation mechanism of the cellulose 19 crystals and highly ordered structure has long been unclear. Considering the specific density of 20 cellulose versus water, the formation of crystalline and highly ordered structure in an aqueous 21 solution should be affected by gravity. Thus, we synthesized cellulose with $C t C D P$ at the 22 International Space Station, where sedimentation and convection due to gravity are negligible. 23 Optical microscopic observation suggested that cellulose in space has a gel-like appearance 24 without apparent aggregation, in contrast to cellulose synthesized on the ground. Small-angle X25 ray scattering (SAXS) and wide-angle X-ray scattering (WAXS) indicated that cellulose 26 synthesized in space has a more uniform particle distribution in the $\sim 100 \mathrm{~nm}$ scale region than 27 cellulose synthesized on the ground. Scanning electron microscopy (SEM) showed that both 28 celluloses have a micrometer scale network structure, whereas a fine fiber network was 29 constructed only under microgravity. These results indicate that gravity plays a role in cellulose II 30 crystal sedimentation and the building of network structure, and synthesis in space could play a 31 role in the design of unique materials.

32 Keywords: cellulose, cellodextrin phosphorylase, synthesis in vitro, microgravity

33 Declarations

34 Funding: This work was supported by JSPS KAKENHI Grant Number 19K15884

35 (grant to NS), by a Grant-in-Aid for Innovative Areas from the Japanese Ministry 36 of Education, Culture, Sports, and Technology (MEXT; grant no. 18H05494 to $37 \mathrm{KI}$ ), and the Academy of Finland through research grant SA-FOSSOK [Decision 
38 No. 309384]. K.I. thanks the Finnish Funding Agency for Innovation for the

39 support of the Finland Distinguished Professor Program "Advanced approaches

40 for enzymatic biomass utilization and modification (BioAD)".

42 Conflicts of interest/Competing interests: Not applicable.

43 Code availability: Not applicable

44 Ethics approval: Not applicable

45 Consent to participate: Not applicable

46 Consent for publication: Not applicable

48 Acknowledgments

49 The authors thank Prof. Motomitsu Kitaoka of Niigata University for providing CtCDP gene and

50 Prof. Tomoya Imai of Kyoto University for helpful discussions. The WAXS and SAXS

51 experiments were conducted at the BL8S3 station of Aichi Synchrotron Radiation Center, Aichi

52 Science \& Technology Foundation, Aichi, Japan (Proposal No. 2020D6031). SEM observation

53 was conducted under the supervision of Dr. Satoshi Kimura of the University of Tokyo. We thank

54 Confocal Sciences Inc. and Japan Manned Space Systems Corporation (JAMSS) for sample

55 preparation and launch to the ISS.

56

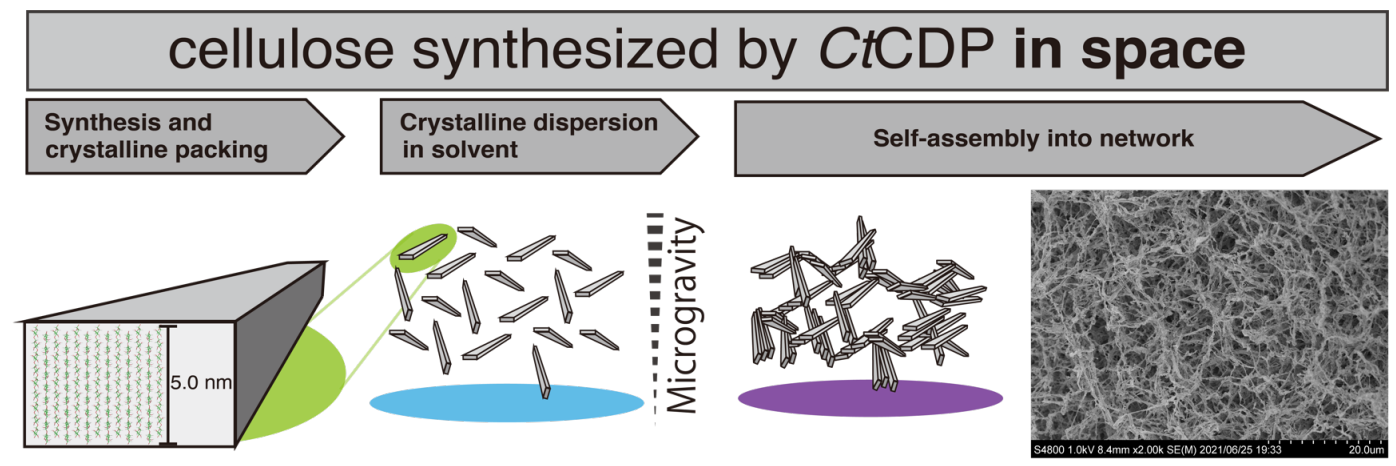

cellulose synthesized by CtCDP on the ground

Synthesis and crystalline packing
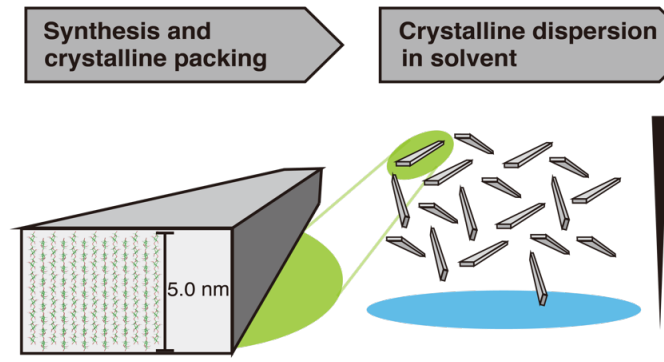
in solvent

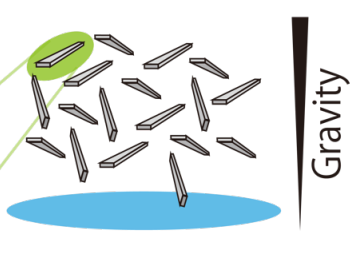

58

Graphical Abstract
Aggregation during self-assembly

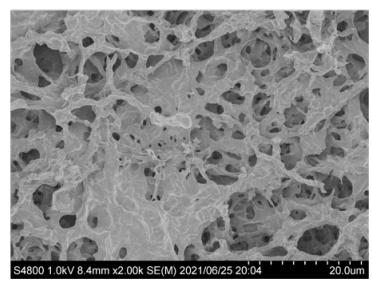




\section{Introduction}

61 Cellulose is the most abundant carbohydrate on Earth, and has been utilized by

62 humans from ancient times. In nature, cellulose is mostly produced by woody and

63 herbaceous plants as a cell-wall component. It is also synthesized by some

64 microorganisms such as Komagataeibacter xylinus (Acetobacter xylinum),

65 invertebrate animals (urochordates), or green algae (Cladophora species)

66 (VanderHart and Atalla 1984; Belton et al. 1989; Larsson et al. 1997). Cellulose is

67 a linear polymer of exclusively $\beta$-1,4-glycosidic-bonded glucose molecules

68 synthesized by cellulose synthase complex on the cell membrane of these species.

$69 \beta-1,4-G l u c a n$ chains synthesized by the complex on the cell membrane

70 spontaneously assemble and crystallize to form cellulose microfibrils (CMF; also

71 called cellulose nanofibers, CNF). The shape of CMF depends on the geometry

72 and morphology of the cellulose synthase complex (Brown 1996; Saxena and

73 Brown 2005), but the mechanism of CMF formation is still unknown. Inside

$74 \mathrm{CMF}$, the $\beta$-1,4-glucan chains are bound together by hydrogen bonds and

75 hydrophobic interaction to form a specific crystalline structure. Cellulose $I_{\alpha}$ and $I_{\beta}$

76 are the smallest crystalline units of natural cellulose, and these two natural

77 crystalline allomorphs are composed of glucan chains in parallel orientation

78 (Atalla and VanderHart 1984; Nishiyama et al. 2002, 2003). In contrast, cellulose

79 II is a non-natural crystalline form originally found in mercerized and regenerated

80 cellulose. The crystalline structure of cellulose II is significantly different from

81 those of natural cellulose $\mathrm{I}_{\alpha}$ and $\mathrm{I}_{\beta}$, having an anti-parallel orientation of cellulose

82 molecules (Kolpak and Blackwell 1976; Langan et al. 1999; Kim et al. 2006).

83 Cellulose II may be thermodynamically more stable, considering that it is formed

84 in preference to metastable cellulose $I_{\alpha}$ or $I_{\beta}$ when dissolved $\beta$-1,4-glucan chains

85 are recrystallized.

86 To elucidate the formation mechanism of CMF and to develop new 87 materials applications, synthesis of various forms of artificial cellulose has been 88 attempted (Uryu et al. 1983, 1985; Nakatsubo et al. 1996). Early efforts showed 89 poor regio- and stereo-selectivity, and thus highly substrate-selective enzymatic 90 approaches were adopted (Kobayashi et al. 1991, 2000; Kobayashi and Shoda 91 1995; Kobayashi 2005; Tanaka et al. 2007). Cellodextrin phosphorylase (CDP) is 92 one of the enzymes utilized for the synthesis of cellulose in vitro. Although CDP 93 catalyzes phosphorolysis of cellodextrin (cellooligosaccharide), it is possible to 
94 synthesize cellulose via the reverse reaction by using high concentrations of $\alpha$-D-

95 glucose-1-phosphate $(\alpha-\mathrm{G} 1 \mathrm{P})$ as a glycosyl donor, with glucose and cellobiose as

96 primary glycosyl acceptors (Alexander 1968; Sheth and Alexander 1969;

97 Krishnareddy et al. 2002). The glycosyl donors form $\beta$-1,4-glycoside bonds with

98 the non-reducing ends of glycosyl acceptors. In this manner, platelet lamellae of

99 crystalline cellulose having the degree of polymerization (DP) 9 were formed in

100 vitro (Hiraishi et al. 2009). All these studies aiming to synthesize cellulose in vitro

101 afforded cellulose II. Pylkkänen et al. have found that concentrated cellulose II

102 synthesized by CDP from Clostridium (Hungateiclostridium) thermocellum

103 (CtCDP) formed crystalline platelet lamellae and ribbon-like higher-ordered

104 network structure (Pylkkänen et al. 2020). However, the mechanism of

105 formation of cellulose II's supermolecular structure is still unknown, as is that of

106 natural cellulose $\mathrm{I}_{\alpha}$ and $\mathrm{I}_{\beta}$.

107 Protein crystallization in space enhances the quality of protein crystals due

108 to decreased sedimentation and convection under microgravity (Vekilov 1999).

109 This affords more orderly crystals than can be obtained on the ground, enabling

110 researchers to obtain higher-quality X-ray diffraction data (Snell et al. 1995; Inaka

111 et al. 2011; Nakamura et al. 2015; Tachioka et al. 2017; Yamaguchi et al. 2021).

112 A crystal of alloy semiconductor grown on the International Space Station (ISS)

113 also showed better quality than one grown on the ground (Inatomi et al. 2015),

114 and an $\mathrm{NaCl}$ crystal grown on the ISS had different morphology from a crystal

115 grown on Earth (Fontana et al. 2011). On the other hand, the synthesis and crystal

116 formation of organic polymers such as cellulose under microgravity in space have

117 not yet been investigated.

118 In the present study, cellulose II was synthesized in vitro using CtCDP on

119 the ISS. We investigated how gravity affects cellulose II crystalline or higher-

120 order structure formation by comparing the product with material synthesized in

121 the same way on the ground, employing small-angle x-ray scattering (SAXS),

122 wide-angle X-ray scattering (WAXS), and scanning electron microscopy (SEM). 


\section{Materials and Methods}

125 Materials.

$126 \alpha-G 1 P$ and pET-28b vector were purchased from Sigma-Aldrich Co. LLC (MO, 127 US). Cellobiose and other chemical reagents were purchased from FUJIFILM 128 Wako Pure Chemical Corporation (Osaka, Japan). Overnight Express auto129 induction medium and BugBuster reagents were purchased from Merck KGaA 130 (Darmstadt, Germany). CDP from Clostridium (Hungateiclostridium) 131 thermocellum strain YM4 was initially provided by Prof. Momomitsu Kitaoka of 132 Niigata University, Japan. E. coli BL21 (DE3) competent cells were purchased 133 from Nippon Gene (Tokyo, Japan). C-Tube counter-diffusion (Otálora et al. 2009) 134 quartz capillaries were purchased from Confocal Sciences Inc. (Tokyo, Japan).

\section{Enzyme preparation.}

136 A gene coding $\triangle \mathrm{Cys}-C t \mathrm{CDP}$ based on CDP from $C$. thermocellum strain YM4 137 (GenBank: AB061316.1) was designed, in which all 11 cysteine residues were 138 replaced with serine residues. None of the cysteine residues in $C t \mathrm{CDP}$ are thought 139 to form disulfide bonds. This gene was codon-optimized for expression in E. coli 140 and synthesized by GenScript (NJ, US) with a 6x His tag at the C-terminal. It was 141 inserted into the pET-28b vector between the NcoI and XhoI sites with Ligation 142 High (Toyobo, Osaka, Japan). The vector was transformed into E. coli BL21 143 (DE3). $\triangle$ Cys-CtCDP was expressed while transformed cells were cultivated in an

144 Erlenmeyer flask filled with $1 \mathrm{~L}$ of Overnight Express auto-induction medium at $14530^{\circ} \mathrm{C}$. After 18 hours of cultivation, the cells were collected by centrifugation, and 146 the crude enzyme was obtained after cell lysis with BugBuster reagents. The 147 crude enzyme was purified on a TALON his-tag cobalt affinity column (Clontech 148 Takara Bio USA, CA, US). The His-tagged target protein was eluted with a linear 149 gradient of $20 \mathrm{mM}$ Tris- $\mathrm{HCl}$ buffer $\mathrm{pH} 7.5$ containing $100 \mathrm{mM} \mathrm{NaCl}$ and $500 \mathrm{mM}$ 150 imidazole. The His-tagged protein was then dialyzed against $20 \mathrm{mM}$ Tris- $\mathrm{HCl}$ 151 buffer with an Amicon apparatus with 10,000 MWCO Biomax membrane filter 152 (Merck). Anion exchange chromatography with TOYOPEARL DEAE-650S 153 (Tosoh, Tokyo, Japan) was employed for further purification. Highly purified $154 \Delta$ Cys-CtCDP was eluted with a linear gradient of $20 \mathrm{mM}$ Tris-HCl buffer $\mathrm{pH} 7.5$ 155 containing $250 \mathrm{mM} \mathrm{NaCl}$ and used for cellulose synthesis. 
$1570.10 \mu \mathrm{g} / \mathrm{ml} \Delta$ Cys-CtCDP and $10 \mathrm{mM}$ cellobiose were introduced into a C-tube 158 counter-diffusion (Otálora et al. 2009) quartz capillary placed in $10 \mathrm{mM}$ 159 cellobiose and $200 \mathrm{mM} \alpha-\mathrm{G} 1 \mathrm{P}$ solution three days before launch. The counter160 diffusion capillary consists of a $2 \mathrm{~mm}$ diameter quartz capillary and silicon tubing

161 containing agarose gel; this arrangement allows the outer solution to diffuse into 162 the capillary. Inside the counter-diffusion capillary, the initial $\alpha$-G1P 163 concentration was set to $0 \mathrm{mM}$ and this gradually increased as $\alpha$-G1P diffused 164 from the gel tube (Fig. 1A). The $\alpha$-G1P concentration was controlled to minimize 165 the influence of gravity during cellulose synthesis before arrival at the ISS. The 166 Kirara service (JAMSS, Tokyo, Japan) was used to launch the experiment to the 167 ISS. The sample was kept in the microgravity environment of the ISS for one 168 month at $20^{\circ} \mathrm{C}$ inside a thermostated box (Fig. 1B). The cellulose synthesized on 169 Earth was prepared similarly, except for the presence of gravity, as a control.

170 WAXS measurements.

171 WAXS measurements were done at the BL8S3 station of Aichi Synchrotron 172 Radiation Center (Aichi, Japan) with a $205.85 \mathrm{~mm}$ camera length. R-Axis IV++ 173 (Rigaku, Tokyo, Japan) was used to record the diffraction, and radial integration 174 of diffraction intensity was performed with the program FIT2D (ESRF, Grenoble, 175 France). Sample capillaries were attached to the cell holder, and measurements 176 were conducted at the upper part (10 $\mathrm{mm}$ from the capillary top), middle part (14 $177 \mathrm{~mm}$ from the capillary top), and bottom part (18 $\mathrm{mm}$ from the capillary top) of the 178 capillary (Fig. 1C).

179 Igor Pro (Wavemetrics, OR, US) was used to perform WAXS peak fit 180 analysis and to create graphics. FWHM (full width at half maximum) of peaks 181 assigned to the 020 plane of cellulose II and peak areas were determined, 182 assuming that scattering due to water was smooth and would not form any peak.

\section{SAXS measurements.}

184 SAXS experiment was conducted at the BL8S3 station of Aichi Synchrotron 185 Radiation Center under the following conditions: diffraction of $0.92 \AA$ X-rays was 186 recorded on an R-Axis IV++ at a camera length of $3975.85 \mathrm{~mm}$. Radial 187 integration of diffraction intensity was performed with the program FIT2D. 
188 Sample capillaries were attached to the cell holder, and measurements were done 189 at the upper part (10 $\mathrm{mm}$ from the capillary top), middle part (14 $\mathrm{mm}$ from the 190 capillary top), and bottom part (18 $\mathrm{mm}$ from the capillary top) of each sample 191 capillary (Fig. 1C).

192 SAXS data was processed with ATSAS (Manalastas-Cantos et al. 2021) and 193 SasView (http://www.sasview.org/). The SAXS data were analyzed after

194 subtracting the scattering curve of the negative control solution containing 0.10

$195 \mu \mathrm{g} / \mathrm{ml} \mathrm{CtCDP}$ and $10 \mathrm{mM}$ cellobiose in a C-tube capillary. Earlier electron 196 microscopy and atomic microscopy observations showed that complex structural 197 features can co-exist in one reaction system (Hiraishi et al. 2009; Pylkkänen et al. 198 2020), and therefore we used a unified power law equation for fitting the data 199 (Beaucage 1995; Tajima et al. 2019).

$I(Q)=$ background $+\sum_{i=1}^{2}\left[G_{i} \cdot \exp \left(-\frac{Q^{2} \cdot R g_{i}^{2}}{3}\right)+B_{i} \cdot \exp \left(-\frac{Q^{2} \cdot R g_{i+1}^{2}}{3}\right)\right.$.

$\left.202\left(\frac{1}{Q_{i}^{*}}\right)^{P_{i}}\right]$

$$
Q_{i}^{*}=Q\left[\operatorname{erf}\left(\frac{Q \cdot R g_{i}}{\sqrt{6}}\right)\right]^{-3}
$$

205 Q, I(Q), Rg, G, B in equations (1) and (2) are scattering vector, intensity, radius of 206 gyration for a particular scattering body, Guinier function, and Porod-type 207 function, respectively. The scattering vector was defined as $Q=4 \pi / \lambda \sin \theta$, where $2082 \theta$ is the scattering angle, and $\lambda$ is the wavelength.

209 Observation with scanning electron microscopy (SEM).

210 The solvent in capillaries containing synthesized cellulose was replaced gradually 211 with tert-butyl alcohol and then the cellulose samples were freeze-dried in a 212 lyophilizer (FDU-1200, Eyela, Tokyo, Japan) and collected by breaking the 213 capillaries with a cutting stone (Hampton Research, CA, US). Samples were 214 coated with Pt-Pd, and SEM images were captured with an FE-SEM S-4800 215 (Hitachi, Tokyo, Japan) at $1 \mathrm{kV}$. 


\section{Results and discussion}

\section{Enzyme preparation}

219 CtCDP was found to be unstable and lost its activity over several weeks. Since

220 synthesis of cellulose on the ISS was planned for one month, improving the

221 stability of $C t$ CDP was the first challenge for this study. Alexander et al.

222 suggested that the oxidation state of cysteine residues negatively affects the

$223 C t$ CDP activity, and therefore, we designed $\Delta$ Cys-CtCDP in which all 11 cysteine

224 residues are replaced with serine residues (Fig. 2). This $\Delta$ Cys-CtCDP did not lose

225 activity for at least two months. Characterization of the mutated CtCDP will be

226 reported elsewhere.

228 Optical observation of cellulose synthesized in counter-diffusion 229 capillaries

230 In the $C t C D P$ reaction using the counter-diffusion reaction vessel, the reaction 231 proceeds as the donor substrate, $\alpha-\mathrm{G} 1 \mathrm{P}$, is supplied from the gel tube by diffusion 232 (Fig. 1A). In the sample capillaries, there was an unreacted region, where no 233 product exists, on the opposite side from the gel tube. This result suggests that the 234 enzymatic reaction proceeded sequentially from the site of the gel tube, regardless 235 of whether the reaction takes place in space or on the ground (Fig 3A and B).

236 However, the appearance of the cellulose synthesized under the two conditions 237 differed significantly.

238 The cellulose synthesized on the ISS had an overall homogeneous gel-like 239 appearance, and no aggregates could be seen (Fig 3A). However, on the ground, 240 the formation of larger aggregates was observed, and they were more abundant 241 near the base of the gel tube, i.e., in the direction of gravity (Fig 3B). The density 242 of cellulose crystals is approximately $1.5 \mathrm{~g} / \mathrm{cm}^{3}$, and under typical aqueous 243 reaction conditions, the synthesized cellulose particles be expected to settle under 244 gravity. This settling would not occur in the microgravity environment in space, 245 suggesting that cellulose synthesis under microgravity prevents the formation of 246 visible highly ordered structures and aggregates, affording more homogeneous 247 cellulose crystals. In addition, the highly ordered structure of cellulose 
248 synthesized under microgravity was sufficiently strong to withstand its weight

249 because no aggregation was observed after the return to the Earth.

\section{WAXS measurements}

252 The cellulose synthesized in space appeared homogeneous and gel-like. On the

253 other hand, it is known that the crystalline form of cellulose is affected by drying

254 and other factors, so it was necessary to leave the cellulose in the reaction 255 capillary to perform X-ray diffraction measurements. To identify the allomorphs 256 of cellulose synthesized under microgravity and on the ground, WAXS diffraction 257 measurements were conducted. The WAXS diagram is shown in Fig. 4. The 258 scattering intensity increased monotonically in the range of $5 \mathrm{~nm}^{-1}<\mathrm{Q}<16 \mathrm{~nm}^{-1}$ 259 due to the presence of an excess amount of water. All measurements showed 260 similar trends (Fig. 4). However, the scattering intensities of cellulose synthesized 261 in space were similar along the height direction of the capillary, in contrast to the 262 scattering intensities of cellulose synthesized on the ground, where the upper part 263 showed higher scattering intensity in all ranges $\left(5 \mathrm{~nm}^{-1}<\mathrm{Q}<16 \mathrm{~nm}^{-1}\right)$. This 264 suggests that cellulose synthesized in space has a more uniform crystal size or 265 more uniform crystal orientation in the height direction of the capillary than 266 cellulose synthesized on the ground.

267 As shown in Fig. 4, three peaks were detectable in the range of $5 \mathrm{~nm}^{-1}<\mathrm{Q}<$ $26816 \mathrm{~nm}^{-1}$. There were weak peaks in the WAXS diagram of cellulose synthesized in 269 space, whereas cellulose synthesized on the ground showed sharp peaks. 270 According to the formula $d=2 \pi / Q$, which describes the relationship between 271 scattering vector $(\mathrm{Q})$ and real space $(\mathrm{d})$, peaks of $\mathrm{Q}=8.69,14.1$, and $15.6 \mathrm{~nm}^{-1}$ 272 correspond to real space $\mathrm{d}=7.23,4.45$ and $4.03 \AA$, respectively. A combination of 273 those d-values was matched with lattice spaces in the 110,110 , and 020 planes of 274 cellulose II, respectively (Kobayashi et al. 2011; French 2014). Therefore, 275 celluloses synthesized on the ground and under microgravity were both assigned 276 as crystalline cellulose II. Thus, gravity did not appear to influence the 277 polymorphic form of the prooduct.

278 The areas and FWHMs of peaks attributed to the 020 plane in Fig. 4 were 279 determined and are summarized in Table 1. The 020 plane areas of ground280 synthesized cellulose II were larger than those of space-synthesized cellulose. The 
281 average peak area of cellulose on the ground was twice as large as that of 282 cellulose synthesized in space, and the average FWHM was $10 \%$ smaller. Those 283 data suggest that cellulose synthesized in space has a smaller crystal size or 284 reduced degree of crystal orientation.

\section{SAXS measurements}

287 WAXS measurements confirmed that the cellulose synthesized in space was not 288 an amorphous gel, but consisted of particles of crystalline cellulose. Therefore, 289 SAXS measurements were carried out to obtain information on this particulate 290 cellulose.

291 Fig. 5 shows Log-Absolute SAXS plots and residual plots after subtraction 292 of scattering from the bottom part of each capillary. The residual plots indicate 293 that cellulose synthesized in space showed a more uniform density of particles 294 with various radii of gyration throughout the capillary than cellulose synthesized 295 on the ground (Fig. 5B and D). Specifically, there were more components in the 296 region of $\mathrm{Q}<1.0 \mathrm{~nm}^{-1}$ in the upper and middle parts compared to the bottom part, 297 though there was no significant difference between the plots of the upper and 298 middle parts. Thus, the SAXS profile of cellulose synthesized on the ground 299 differed more depending on the position in the capillary, and the difference was 300 particularly pronounced in the region of $\mathrm{Q}<1.5 \mathrm{~nm}^{-1}$, which means the particle 301 region with a radius of gyration $R_{g}$ greater than $4.18 \mathrm{~nm}$ (Fig. 5C). As for 302 cellulose synthesized on the ground, scattering from the middle part of the 303 capillary was higher than scattering from other parts (Fig. 5C and D). Considering 304 that gravitational settling is the main cause of the variation in particle distribution 305 with capillary position, the observation of a higher density in the middle part of 306 the capillary seems strange. However, it might be explained by adsorption of 307 cellulose II lamellar crystals on the quartz glass during sedimentation and 308 aggregation. CtCDP-cellulose II lamellar crystals have a large hydrophilic area 309 with abundant hydroxyl groups on the surface (Hiraishi et al. 2009; Wada et al. 310 2021), and might therefore bind readily with $\mathrm{SiO}_{2}$ at the surface of the capillary.

311 To further highlight the differences, a Kratky plot was performed (Fig. 6).

312 The scattering from the center of the ground-synthesized cellulose showed a clear 313 peak at $\mathrm{Q} \approx 0.90 \mathrm{~nm}^{-1}$, which is distinctly different from that of space-synthesized 
314 cellulose. While the scattering intensity of cellulose synthesized on the ground 315 varied with the height in the capillary, the scattering intensity of cellulose 316 synthesized under microgravity was relatively homogeneous. These data 317 qualitatively suggest that there was no significant difference in the number and 318 volume of cellulose particle scatterers in the upper or bottom part of the capillary 319 between the ground and space conditions. Nevertheless, there was a significant 320 difference in the number and volume of scatterers in the middle part.

321 To quantitatively evaluate the size of the scatterers, we focused on the 322 small-angle results in the SAXS measurements. We found that a unified power 323 law equation (Beaucage 1995) gave a good fit, with sufficiently small values of $324 \mathrm{chi}^{2} /$ point for all parts of the capillaries (Fig. 7). Especially in the region of 0.07 $325 \mathrm{~nm}^{-1}<\mathrm{Q}<0.5 \mathrm{~nm}^{-1}$, all SAXS scatterings were proportional to $\mathrm{Q}^{-2.28}-\mathrm{Q}^{-2.35}$, 326 indicating that the particles have a thin plate shape, whether the cellulose is 327 synthesized in space or on the ground (Kratky and Porod 1949; Pedersen 1997).

328 This conclusion is consistent with previous studies showing that CtCDP-cellulose 329 single crystals have a platelet shape (Hiraishi et al. 2009; Pylkkänen et al. 2020; 330 Wada et al. 2021).

331 The small-angle region of the SAXS results did not show a good fit in 332 Guinier plot analysis for all the samples. This suggests that all the samples 333 obtained consist of a set of aggregates with multiple radii of gyration. Therefore, 334 in this fitting analysis, we focused only on the radius of gyration $\mathrm{R}_{\mathrm{g} 2}$, which 335 corresponds to the peak at $\mathrm{Q} \approx 0.9 \mathrm{~nm}^{-1}$. Table 2 shows all the parameters of the 336 fitting analysis; the average $\mathrm{R}_{\mathrm{g} 2}$ values for cellulose in space and on the ground 337 were calculated to be $6.61 \pm 0.09 \mathrm{~nm}$ and $4.57 \pm 0.84 \mathrm{~nm}$, respectively. It has been 338 shown that cellulose synthesized in vitro by $C t C D P$ under batch conditions on the 339 ground has a degree of polymerization of 9 and forms plate-like crystals with a 340 thickness of about $5 \mathrm{~nm}$ (Hiraishi et al. 2009). This value is similar to the $\mathrm{R}_{\mathrm{g} 2}$ 341 values of cellulose in space and on the ground. Thus, CtCDP-cellulose's 342 crystalline lamellar structure existed in celluloses synthesized both in space and 343 on the ground.

344 The parameter $\mathrm{B}_{2}$ in Table 2 represents the number or density of particles 345 having a radius of gyration $\mathrm{R}_{\mathrm{g} 2}$. Cellulose synthesized in space had uniform $\mathrm{B}_{2}$ 346 values at all measured points $(1.32,1.34$, and 1.27 for the capillary's upper, 347 middle, and bottom parts, respectively). In contrast, cellulose on the ground had 
348 different values $(1.42,4.18$, and 1.94 for the upper, middle, and bottom parts of 349 the capillary). This difference suggested that cellulose synthesized in space has a 350 quantitatively more uniform density of particles with a radius of gyration $\mathrm{R}_{\mathrm{g} 2}$ in

351 the height direction of capillary, as compared with cellulose on the ground.

\section{Observation with SEM}

354 Typical SEM images are shown in Fig. 8. Cellulose synthesized in space (Fig. 8A 355 and B) had a finer network structure than cellulose synthesized on the ground 356 (Fig. 8C and D). The network consisted of ribbon-like structures, which were 357 estimated to be 100-200 nm wide in space-synthesized cellulose (Fig. 8B). In 358 contrast, cellulose synthesized on the ground contained thicker aggregates with 359 micrometer scale width (Fig. 8D), i.e., several times larger. The width of the thin 360 ribbon-like structures synthesized in space was consistent with previous TEM and 361 AFM observations of CDP-synthesized crystalline cellulose II (Hiraishi et al. 362 2009; Pylkkänen et al. 2020). This indicates that space-cellulose's ribbon-like 363 structure was comprised of single to several cellulose crystals, while ribbons of 364 cellulose synthesized on the ground contained more crystals. These results and the 365 WAXS peak intensities suggest that the thick aggregated form might assemble 366 through orientation or crystallization. These partial features of the network 367 structure are consistent with the sparse (Fig. 8A and B) and dense (Fig. 8C and D) 368 micrometer-scale appearance of cellulose network structure. The scale of these 369 ribbon widths is similar to the scale of the wavelength of the visible light; 370 therefore, these features would affect the optical appearance (Fig. 3) as well.

371 It is well known that cellulose II synthesized by CtCDP self-assembles into 372 a network structure (Pylkkänen et al. 2020). Such cellulose II synthesized by $373 C t \mathrm{CDP}$ on the ground was observed as white precipitates or aggregates in the 374 earlier studies, in contrast to the gel-like appearance of space-synthesized 375 cellulose (Fig. 3A). In previous attempts to create gel-like products with 376 supermolecular network architecture on the ground, researchers have added 377 nanocrystals of polymers such as polyethylene glycol and cellulose $\mathrm{I}_{\beta}$ to the 378 reaction mixture for cellulose synthesis by $C t C D P$ (Hata et al. 2017, 2018) to 379 serve as scaffolds. We believe the present report is the first to describe the 380 production of pure cellulose II crystalline gel. 
381 Our observations indicate that once the cellulose II network structure is 382 formed in space, the supermolecular structure is stable after return to Earth. A 383 relatively light polymer (PMMA, $1.2 \mathrm{~g} / \mathrm{cm}^{3}$ ) was reported to form a network 384 structure of crystalline polymer through viscoelastic phase separation on the 385 ground, and gravity appeared to have a negligible influence for at least 12 hours 386 (Tsurusawa et al. 2017). Thus, it is possible that the effect of gravity on cellulose 387 arises because of the high specific gravity of cellulose compared with water. 388 Therefore, the microgravity environment in space may be essential for the 389 production of cellulose II crystalline gel.

\section{Conclusion}

393 In the present study, we investigated the possibility that gravity influences the 394 crystallization and formation of highly ordered structure of cellulose II. We found 395 that cellulose synthesized in space did not form aggregates like those of cellulose 396 synthesized on the ground. WAXS demonstrated that similar nano-scale 397 crystalline cellulose II packing occurred on the ground and in space. However, the 398 SAXS experiment showed that cellulose particles in a capillary had higher 399 homogeneity when synthesized in space. SEM observation showed that space400 synthesized cellulose had a fine supramolecular network structure on the 401 micrometer scale, and this was strong enough to survive after return to Earth. 402 These findings suggest that gravity influences aggregate formation during self403 assembly to form the network. In this work, a bottom-up synthesis of pure 404 cellulose II crystal gel was achieved for the first time. The physical properties of 405 this newly created cellulose II crystalline gel remain to be investigated. 


\section{References}

408 Alexander JK (1968) Purification and specificity of cellobiose phosphorylase 409 from Clostridium thermocellum. J Biol Chem 243:2899-2904. 410 https://doi.org/10.1016/s0021-9258(18)93356-9

411 Atalla RH, VanderHart DL (1984) Native cellulose: A composite of two distinct 412 crystalline forms. Science 223:283-285.

413 https://doi.org/10.1126/science.223.4633.283

414 Beaucage G (1995) Approximations Leading to a Unified Exponential/Power415 Law Approach to Small-Angle Scattering. J Appl Crystallogr 28:717-728. 416 https://doi.org/10.1107/s0021889895005292

417 Belton PS, Tanner SF, Cartier N, Chanzy H (1989) High-Resolution Solid-State $418{ }^{13} \mathrm{C}$ Nuclear Magnetic Resonance Spectroscopy of Tunicin, an Animal Cellulose. 419 Macromolecules 22:1615-1617. https://doi.org/10.1021/ma00194a019

420 Brown RM (1996) The biosynthesis of cellulose. J Macromol Sci - Pure Appl 421 Chem 33:1345-1373. https://doi.org/10.1080/10601329608014912

422 Fontana P, Schefer J, Pettit D (2011) Characterization of sodium chloride crystals 423 grown in microgravity. J Cryst Growth 324:207-211. 424 https://doi.org/10.1016/j.jcrysgro.2011.04.001

425 French AD (2014) Idealized powder diffraction patterns for cellulose polymorphs.

426 Cellulose 21:885-896. https://doi.org/10.1007/s10570-013-0030-4

427 Hata Y, Kojima T, Koizumi T, et al (2017) Enzymatic Synthesis of Cellulose 428 Oligomer Hydrogels Composed of Crystalline Nanoribbon Networks under 429 Macromolecular Crowding Conditions. ACS Macro Lett 6:165-170. 430 https://doi.org/10.1021/acsmacrolett.6b00848

431 Hata Y, Sawada T, Sakai T, Serizawa T (2018) Enzyme-Catalyzed Bottom-Up 432 Synthesis of Mechanically and Physicochemically Stable Cellulose Hydrogels for 433 Spatial Immobilization of Functional Colloidal Particles. Biomacromolecules 434 19:1269-1275. https://doi.org/10.1021/acs.biomac.8b00092

435 Hiraishi M, Igarashi K, Kimura S, et al (2009) Synthesis of highly ordered 436 cellulose II in vitro using cellodextrin phosphorylase. Carbohydr Res 344:2468437 2473. https://doi.org/10.1016/j.carres.2009.10.002

438 Inaka K, Takahashi S, Aritake K, et al (2011) High-quality protein crystal growth 439 of mouse lipocalin-type prostaglandin D synthase in microgravity. Cryst Growth 440 Des 11:2107-2111. https://doi.org/10.1021/cg101370v 
441 Inatomi Y, Sakata K, Arivanandhan M, et al (2015) Growth of InxGa1 - xSb

442 alloy semiconductor at the international space station (ISS) and comparison with

443 terrestrial experiments. npj Microgravity 1:1.

444 https://doi.org/10.1038/npjmgrav.2015.11

445 Kim NH, Imai T, Wada M, Sugiyama J (2006) Molecular directionality in 446 cellulose polymorphs. Biomacromolecules 7:274-280.

447 https://doi.org/10.1021/bm0506391

448 Kobayashi K, Kimura S, Togawa E, Wada M (2011) Crystal transition from

449 cellulose II hydrate to cellulose II. Carbohydr Polym 86:975-981.

450 https://doi.org/10.1016/j.carbpol.2011.05.050

451 Kobayashi S (2005) Challenge of synthetic cellulose. J. Polym. Sci. Part A Polym.

452 Chem. 43:693-710

453 Kobayashi S, Hobson LJ, Sakamoto J, et al (2000) Formation and structure of 454 artificial cellulose spherulites via enzymatic polymerization. Biomacromolecules 455 1:168-173. https://doi.org/10.1021/bm990010w

456 Kobayashi S, Kashiwa K, Kawasaki T, Shoda SI (1991) Novel Method for 457 Polysaccharide Synthesis Using an Enzyme: The First in Vitro Synthesis of 458 Cellulose via a Nonbiosynthetic Path Utilizing Cellulase as Catalyst. J Am Chem 459 Soc 113:3079-3084. https://doi.org/10.1021/ja00008a042

460 Kobayashi S, Shoda S (1995) Chemical synthesis of cellulose and cello-oligomers 461 using a hydrolysis enzyme as a catalyst. Int J Biol Macromol 17:373-379. 462 https://doi.org/10.1016/0141-8130(96)81849-6

463 Kolpak FJ, Blackwell J (1976) Determination of the Structure of Cellulose II. 464 Macromolecules 9:273-278. https://doi.org/10.1021/ma60050a019

465 Kratky O, Porod G (1949) Diffuse small-angle scattering of x-rays in colloid 466 systems. J Colloid Sci 4:35-70. https://doi.org/10.1016/0095-8522(49)90032-X

467 Krishnareddy M, Kim Y-K, Kitaoka M, et al (2002) Cellodextrin Phosphorylase 468 from Clostridium thermocellum YM4 Strain Expressed in Escherichia coli. J Appl 469 Glycosci 49:1-8. https://doi.org/10.5458/jag.49.1

470 Langan P, Nishiyama Y, Chanzy H (1999) A revised structure and hydrogen-

471 bonding system in cellulose II from a neutron fiber diffraction analysis. J Am 472 Chem Soc 121:9940-9946. https://doi.org/10.1021/ja9916254 
473 Larsson PT, Wickholm K, Iversen T (1997) A CP/MAS ${ }^{13}$ C NMR investigation of 474 molecular ordering in celluloses. Carbohydr Res 302:19-25. 475 https://doi.org/10.1016/S0008-6215(97)00130-4

476 Manalastas-Cantos K, Konarev P V., Hajizadeh NR, et al (2021) ATSAS 3.0: 477 expanded functionality and new tools for small-angle scattering data analysis. $\mathrm{J}$ 478 Appl Crystallogr 54:343-355. https://doi.org/10.1107/s1600576720013412

479 Nakamura A, Ishida T, Kusaka K, et al (2015) "Newton's cradle” proton relay 480 with amide-imidic acid tautomerization in inverting cellulase visualized by 481 neutron crystallography. Sci Adv 1:. https://doi.org/10.1126/sciadv.1500263

482 Nakatsubo F, Kamitakahara H, Hori M (1996) Cationic ring-opening 483 polymerization of 3,6-di-O-benzyl- $\alpha$-D-glucose 1,2,4-orthopivalate and the first 484 chemical synthesis of cellulose. J Am Chem Soc 118:1677-1681. 485 https://doi.org/10.1021/ja953286u

486 Nishiyama Y, Langan P, Chanzy H (2002) Crystal structure and hydrogen487 bonding system in cellulose $\mathrm{I}_{\beta}$ from synchrotron X-ray and neutron fiber 488 diffraction. J Am Chem Soc 124:9074-9082. https://doi.org/10.1021/ja0257319

489 Nishiyama Y, Sugiyama J, Chanzy H, Langan P (2003) Crystal Structure and 490 Hydrogen Bonding System in Cellulose $\mathrm{I}_{\alpha}$ from Synchrotron X-ray and Neutron 491 Fiber Diffraction. J Am Chem Soc 125:14300-14306. 492 https://doi.org/10.1021/ja037055w

493 Otálora F, Gavira JA, Ng JD, García-Ruiz JM (2009) Counterdiffusion methods 494 applied to protein crystallization. Prog Biophys Mol Biol 101:26-37. 495 https://doi.org/10.1016/j.pbiomolbio.2009.12.004

496 Pedersen JS (1997) Analysis of small-angle scattering data from colloids and 497 polymer solutions: Modeling and least-squares fitting. Adv Colloid Interface Sci 498 70:171-210. https://doi.org/10.1016/S0001-8686(97)00312-6

499 Pylkkänen R, Mohammadi P, Arola S, et al (2020) In Vitro Synthesis and Self500 Assembly of Cellulose II Nanofibrils Catalyzed by the Reverse Reaction of 501 Clostridium thermocellum Cellodextrin Phosphorylase. Biomacromolecules 502 21:4355-4364. https://doi.org/10.1021/acs.biomac.0c01162

503 Saxena IM, Brown RM (2005) Cellulose biosynthesis: Current views and 504 evolving concepts. Ann. Bot. 96:9-21 
505 Sheth K, Alexander JK (1969) Purification and properties of beta-1,4-

506 oligoglucan:orthophosphate glucosyltransferase from Clostridium thermocellum. J

507 Biol Chem 244:457-464. https://doi.org/10.1016/s0021-9258(18)94451-0

508 Snell EH, Weisgerber S, Helliwell JR, et al (1995) Improvements in lysozyme

509 protein crystal perfection through microgravity growth. Acta Crystallogr Sect D

510 Biol Crystallogr 51:1099-1102. https://doi.org/10.1107/S0907444995012170

511 Tachioka M, Nakamura A, Ishida T, et al (2017) Production of Large-volume

512 Cellulase Crystals for Visualization of Hydrogen Atoms. Int J Microgravity Sci

513 Appl 34:340108. https://doi.org/10.15011//jasma.34.340108

514 Tajima H, Penttilä PA, Imai T, et al (2019) Observation of in vitro cellulose

515 synthesis by bacterial cellulose synthase with time-resolved small angle X-ray

516 scattering. Int J Biol Macromol 130:765-777.

517 https://doi.org/10.1016/j.ijbiomac.2019.02.167

518 Tanaka H, Koizumi S, Hashimoto T, et al (2007) Self-assembly of synthetic

519 cellulose during in-vitro enzymatic polymerization process as studied by a

520 combined small-angle scattering method. Macromolecules 40:6304-6315.

521 https://doi.org/10.1021/ma070699u

522 Tsurusawa H, Russo J, Leocmach M, Tanaka H (2017) Formation of porous

523 crystals via viscoelastic phase separation. Nat Mater 16:1022-1028.

524 https://doi.org/10.1038/nmat4945

525 Uryu T, Yamaguchi C, Morikawa K, et al (1985) Ring-Opening Polymerization of

526 1,4-Anhydro-2,3,6-tri-O-benzyl- $\alpha$-D-glucopyranose and 1,4-Anhydro-2,3,6-tri-O-

527 benzyl- $\beta$-D-galactopyranose. Macromolecules 18:599-605.

528 https://doi.org/10.1021/ma00146a003

529 Uryu T, Yamanouchi J, Kato T, et al (1983) Selective Ring-Opening

530 Polymerization of Di-O-methylated and Di-O-benzylated 1,4-Anhydro-a-D-

531 ribopyranoses and Structure Proof of Synthetic Cellulose-Type Polysaccharide

$532(1 \rightarrow 4)-\beta$-D-Ribopyranan and $(1 \rightarrow 5)-\alpha$-D-Ribofuranan. J Am Chem Soc

533 105:6865-6871. https://doi.org/10.1021/ja00361a021

534 VanderHart DL, Atalla RH (1984) Studies of Microstructure in Native Celluloses

535 Using Solid-State ${ }^{13} \mathrm{C} \quad$ NMR. Macromolecules 17:1465-1472.

536 https://doi.org/10.1021/ma00138a009

537 Vekilov PG (1999) Protein crystal growth - Microgravity aspects. Adv Sp Res

538 24:1231-1240. https://doi.org/10.1016/S0273-1177(99)00725-5 
539 Wada M, Wakiya S, Kobayashi K, et al (2021) Three-dimensional alignment of

540 cellulose II microcrystals under a strong magnetic field. Cellulose 28:6757-6765.

541 https://doi.org/10.1007/s10570-021-03954-z

542 Yamaguchi S, Sunagawa N, Matsuyama K, Tachioka M (2021) Preparation of 543 large-volume crystal of cellulase under microgravity to investigate the mechanism 544 of thermal stabilization. Int J Microgravity Sci Appl 38:1-12. 545 https://doi.org/10.15011//jasma.38.380103

546

547 

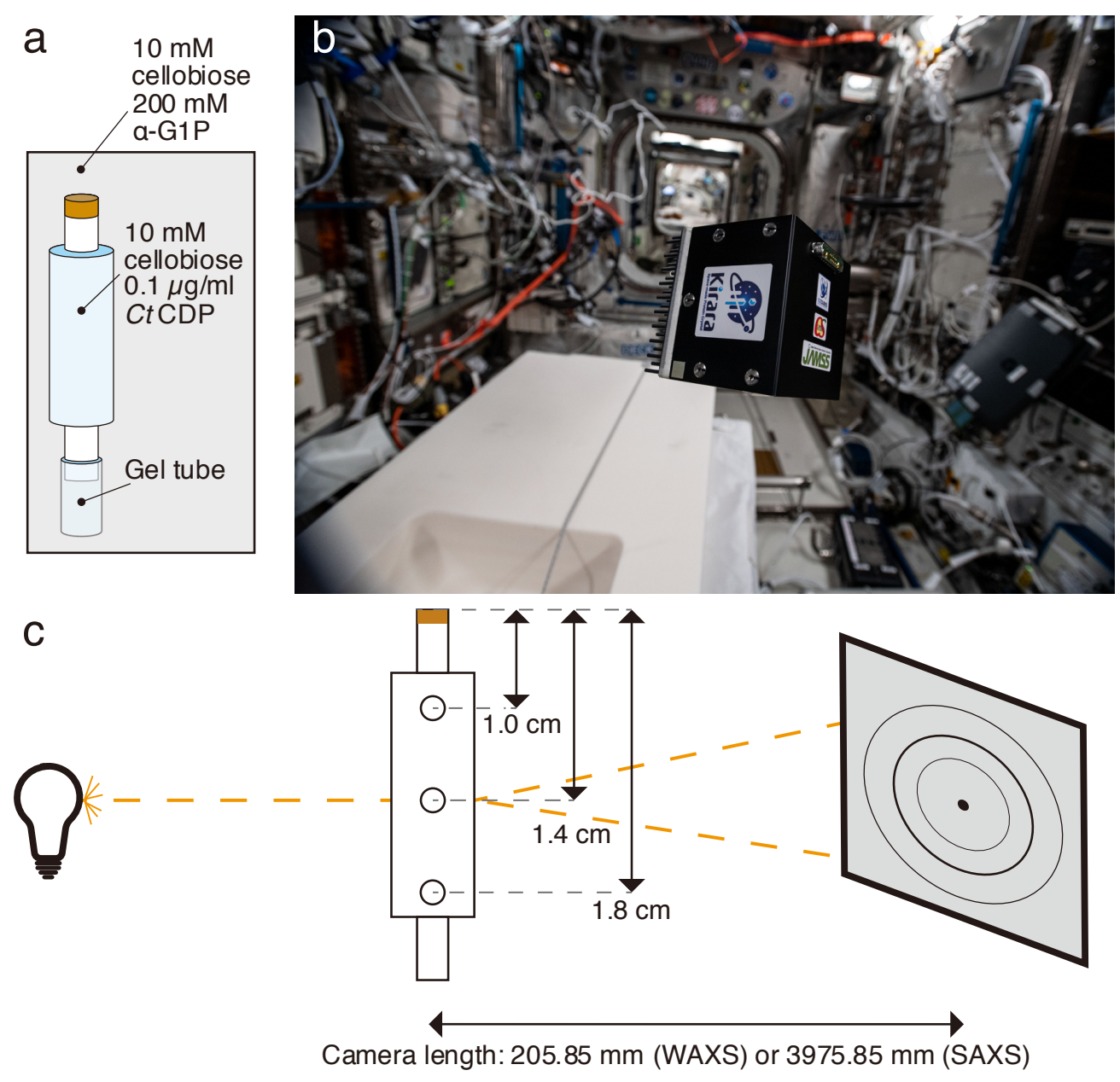

549 Fig. 1. Experimental settings. (a) The counter-diffusion capillary. (b) Thermostated box containing 550 a counter-diffusion capillary for cellulose synthesis under a microgravity environment 551 (CNAS/ESA, Photo was from

552 https://www.esa.int/ESA_Multimedia/Images/2021/01/ICE_Cube_commercial_COVID-

55319 experiment). (c) Schematic illustration of wide-angle and small-angle X-ray scattering 554 experiment on the counter-diffusion capillary. 

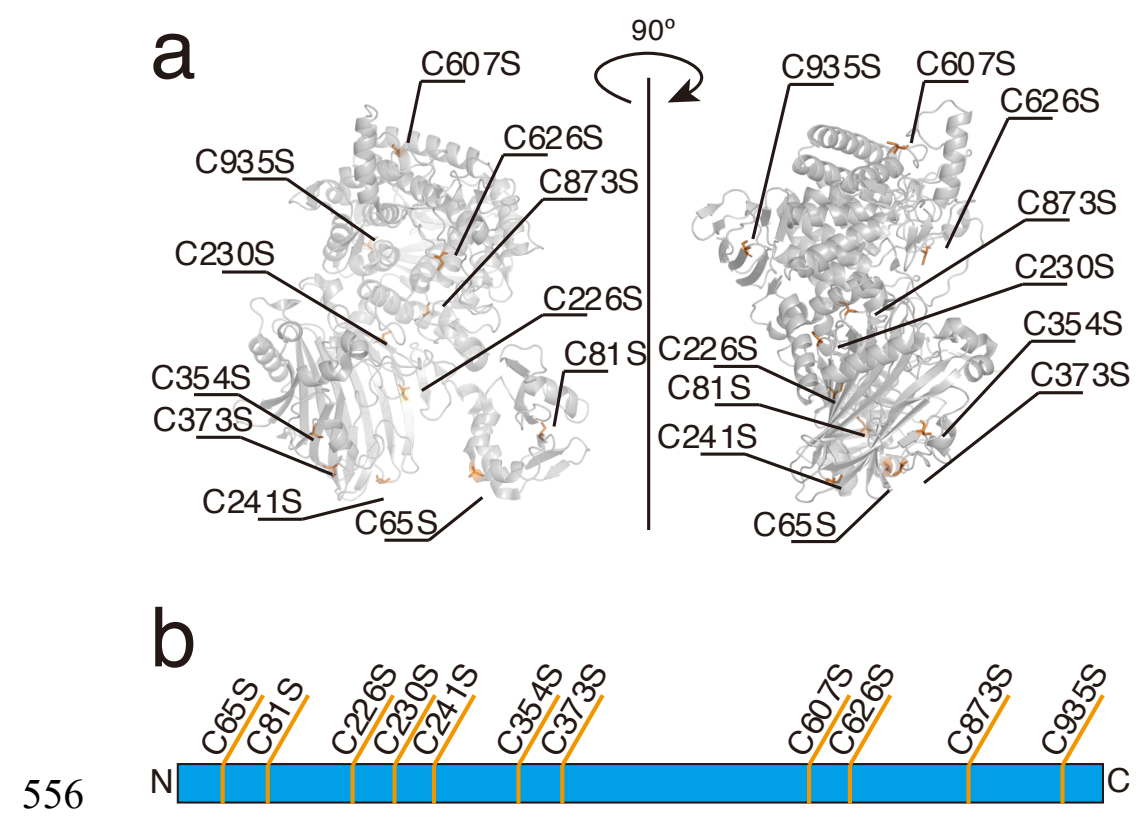

557 Fig. 2. Locations of mutations on $\triangle$ Cys- $C t C D P$ are highlighted in yellow in (a). Images of $C t C D P$ 558 tertiary structure are based on $C t$ CDP monomeric structure (PDB ID: $5 \mathrm{NZ7}$, chain A). The 559 distribution of cysteine-to-serine substitutions in the $C t \mathrm{CDP}$ protein sequence (984 amino acids) is 560 shown in (b). A graphic of the tertiary structure of $C t \mathrm{CDP}$ was created with PyMOL (Schrödinger, 561 NY, US). 

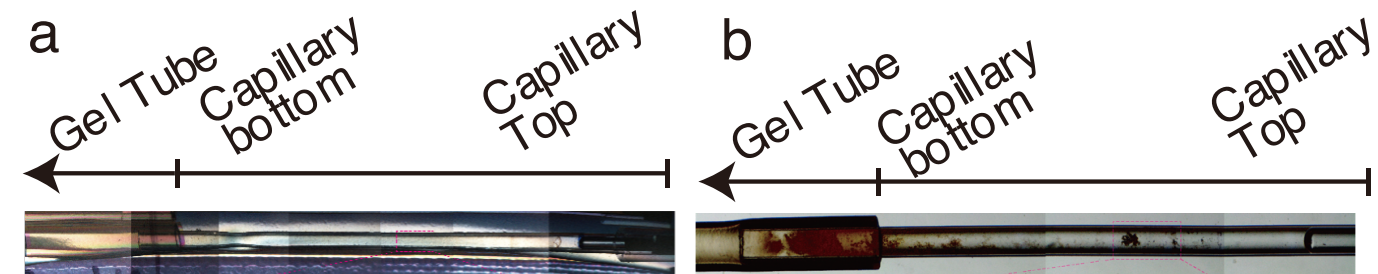

562
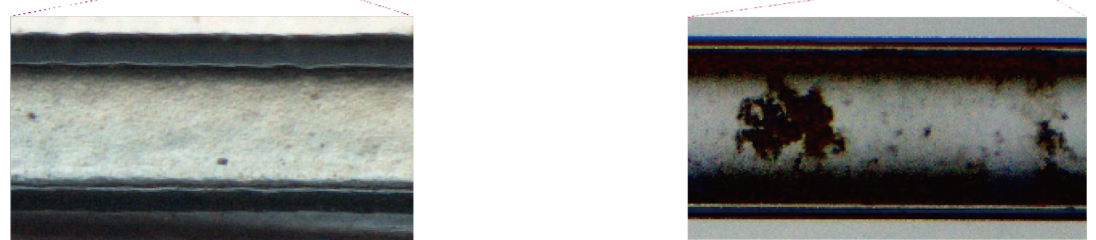

563 Fig. 3. Optical observation of capillaries containing cellulose synthesized in a microgravity 564 environment (a) and on the ground (b). Cellulose synthesized in a microgravity environment 565 showed no apparent aggregation, unlike cellulose synthesized on the ground. 

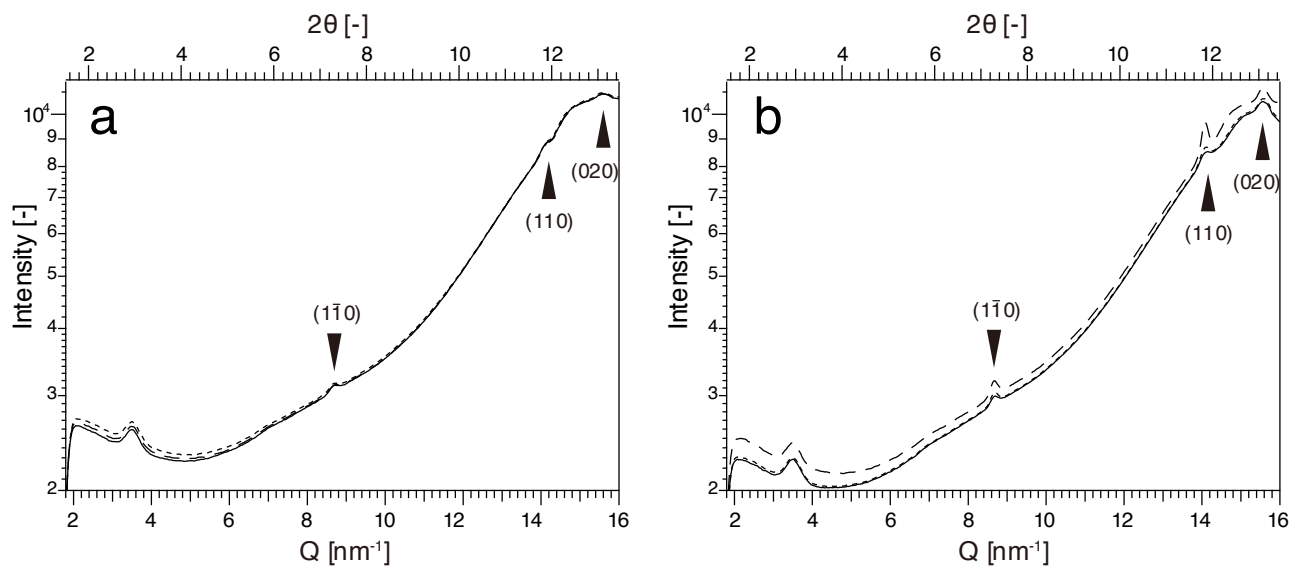

568 Fig. 4. WAXS diagram of cellulose synthesized in a microgravity environment (a) and on the 569 ground (b). Scattering from the upper, middle, and bottom parts are depicted by dashed, dotted, 570 and solid lines, respectively. Cellulose synthesized in a microgravity environment had more 571 uniform and weaker diffraction peaks of cellulose II than cellulose synthesized on the ground.

572 Each arrow shows the location of a peak corresponding to a lattice space of cellulose II. 
a

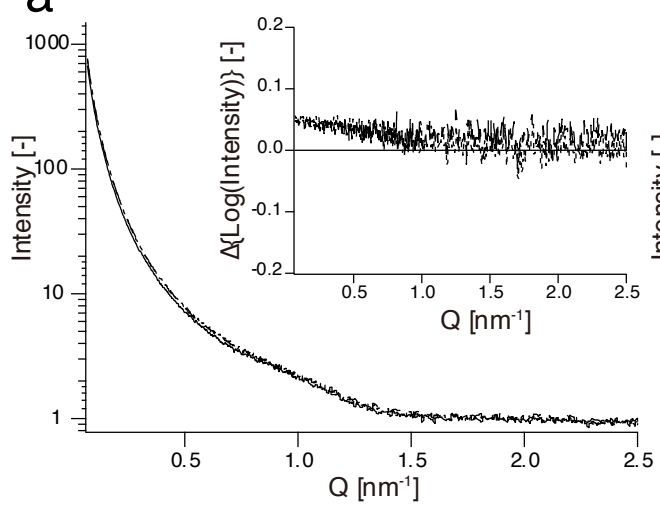

b

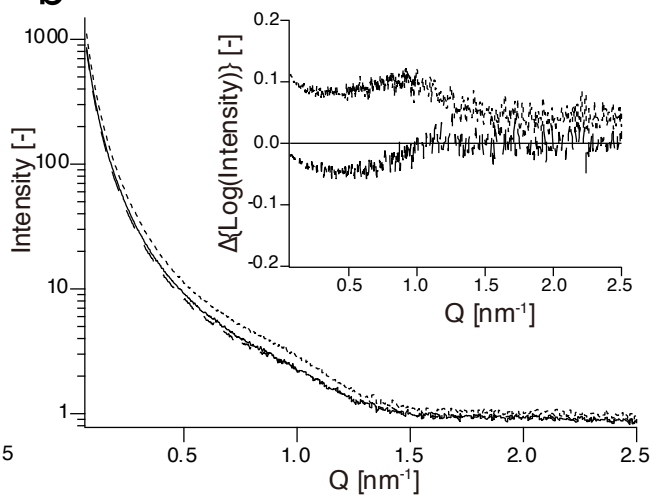

575 Fig. 5. Experimental SAXS curves for $C t C D P-c e l l u l o s e$ and scattering differences in the height 576 direction of the capillaries. SAXS profiles of cellulose synthesized in space and on the ground are 577 shown in (a) and (b), respectively. The insets show residual scattering after subtraction of the 578 scattering from the bottom part of capillaries. Scattering from the upper, middle, and bottom parts 579 are depicted by dashed, dotted, and solid lines, respectively. 


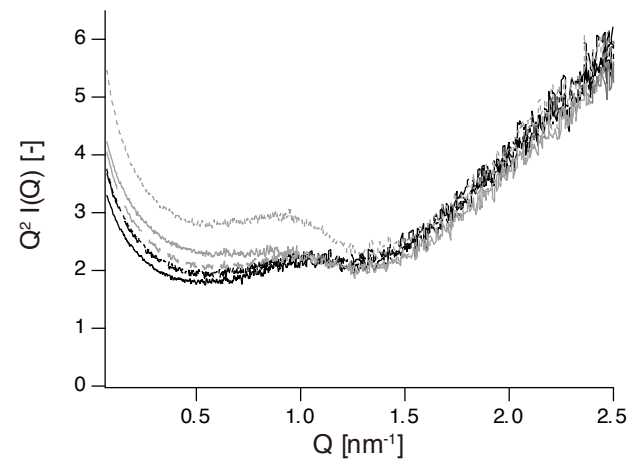

582 Fig. 6. A Kratky plot demonstrating the difference in $C t$ CDP-cellulose particle distribution in the 583 height direction of the capillaries. Kratky plot of cellulose synthesized on the ground (black lines) 584 and in a microgravity environment (gray lines). Scattering from the upper, middle, and bottom 585 parts is depicted by dashed, dotted, and solid lines, respectively. 
a

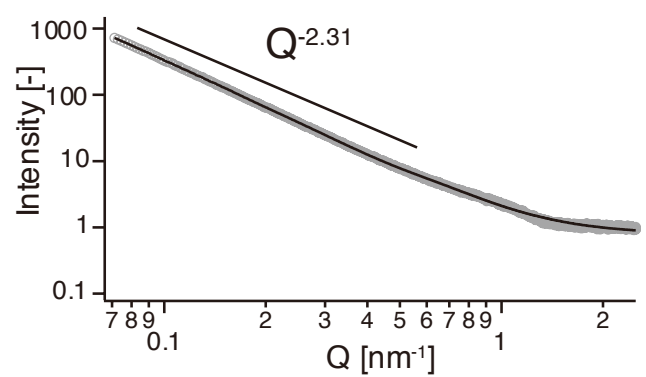

b

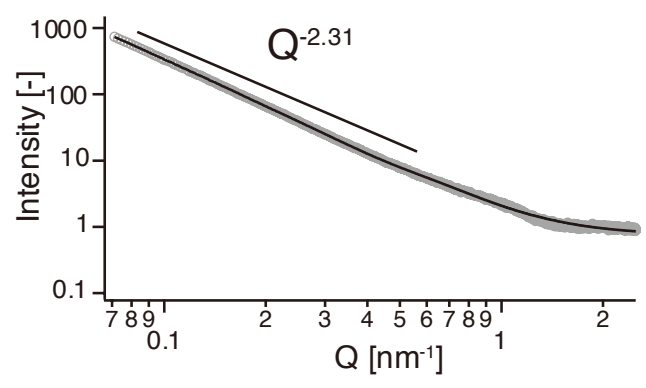

C

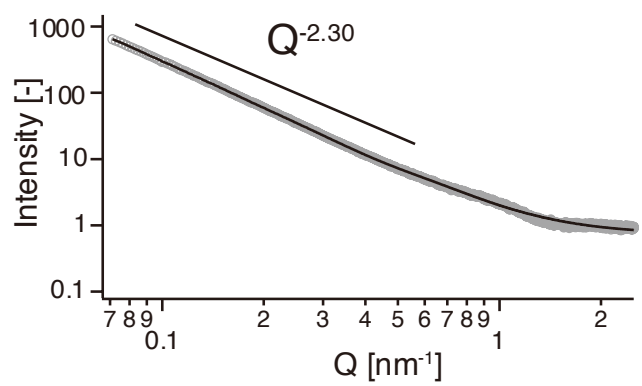

d

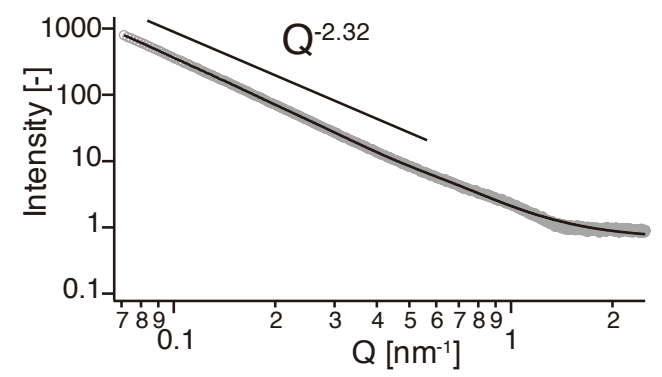

e

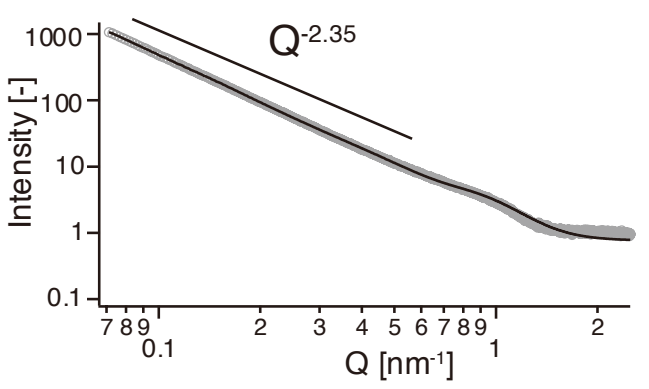

f

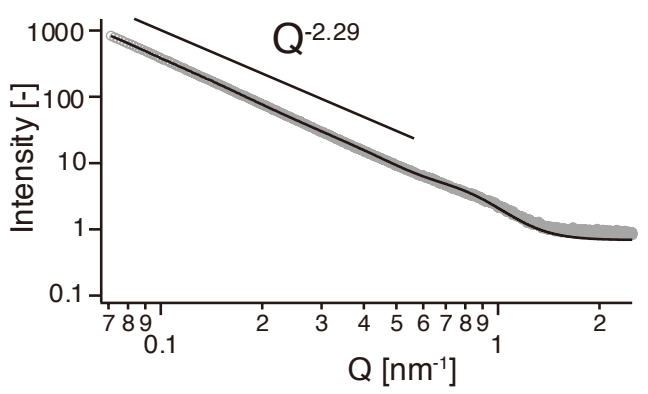

588 Fig. 7. Experimental SAXS profile of $C t$ CDP-cellulose and fitting analysis with equation (1). 589 Scattering of cellulose synthesized in space from the upper part (a), middle part (b), and the 590 bottom part (c) and scattering of cellulose on the ground from the upper part (d), middle part (e), 591 and the bottom part (f) are depicted in log-log plots. Fitted curves are shown as solid lines, and 592 measured values are shown as gray circles in each figure. All SAXS data in the region $0.07 \mathrm{~nm}^{-1}<$ $593 \mathrm{Q}<0.5 \mathrm{~nm}^{-1}$ are proportional to approximately $\mathrm{Q}^{-2.3}$, indicating that the cellulose particles have a 594 platelet shape. 
a

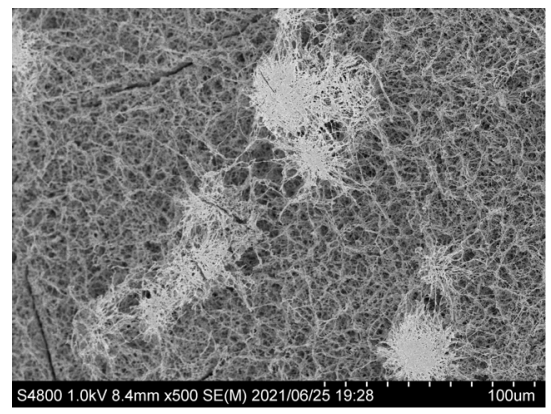

C

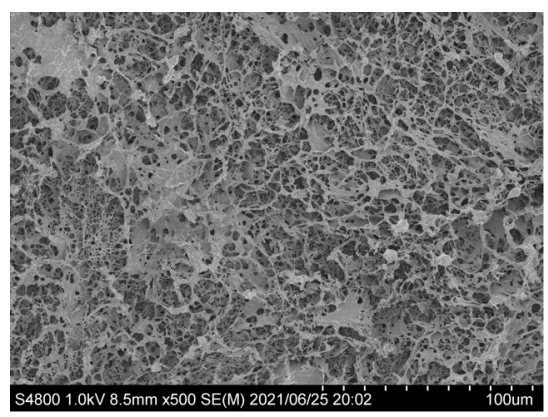

b

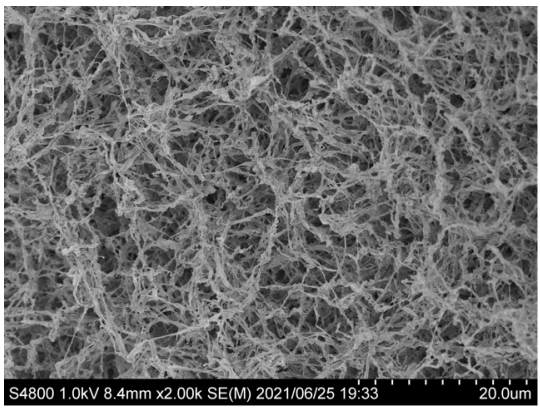

$\mathrm{d}$

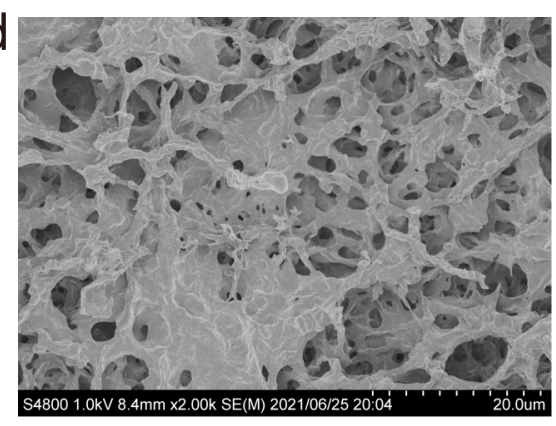

597 Fig. 8. Typical SEM images of cellulose synthesized in space and on the ground. Images of 598 cellulose synthesized in space were captured at x500 (a) and x2000 (b) magnification. (c) and (d) 599 show images of cellulose synthesized on the ground at x500 and x2000 magnification, 600 respectively. Cellulose synthesized under a microgravity environment generated a network 601 consisting of thinner ribbons, while cellulose synthesized on the ground had a network structure 602 with matrix-like thick ribbons. 


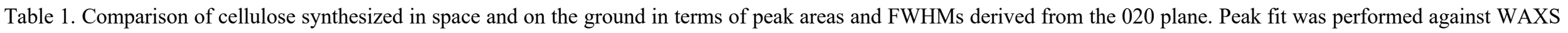

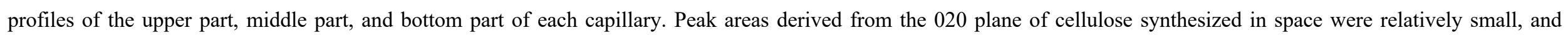
FWHMs were rather large compared to those of cellulose synthesized on the ground. The unit of area is arbitrary.

\begin{tabular}{|l|l|l|l|l|l|l|}
\hline \multicolumn{4}{|l|}{ cellulose synthesized in space } & \multicolumn{3}{l|}{ cellulose synthesized on the ground } \\
\hline & upper part & middle part & bottom part & upper part & middle part & bottom part \\
\hline Area & $125 \pm 34$ & $88.8 \pm 11.7$ & $93.9 \pm 13.9$ & $188 \pm 8$ & $234 \pm 14$ & $246 \pm 20$ \\
\hline $\begin{array}{l}\text { FWHM } \\
\left({ }^{\circ}\right)\end{array}$ & $0.379 \pm 0.055$ & $0.282 \pm 0.025$ & $0.296 \pm 0.029$ & $0.250 \pm 0.008$ & $0.292 \pm 0.011$ & $0.316 \pm 0.015$ \\
\hline $\begin{array}{l}\text { Average } \\
\text { Area }\end{array}$ & 103 & 222 & & \\
\hline $\begin{array}{l}\text { Average } \\
\text { FWHM } \\
\left({ }^{\circ}\right)\end{array}$ & 0.319 & 0.286 & & & \\
\hline
\end{tabular}


Table 2. Parameters for fitting SAXS profiles to equation (1). Fitting analysis was performed for SAXS profiles of the upper part, middle part, and bottom part of each capillary.

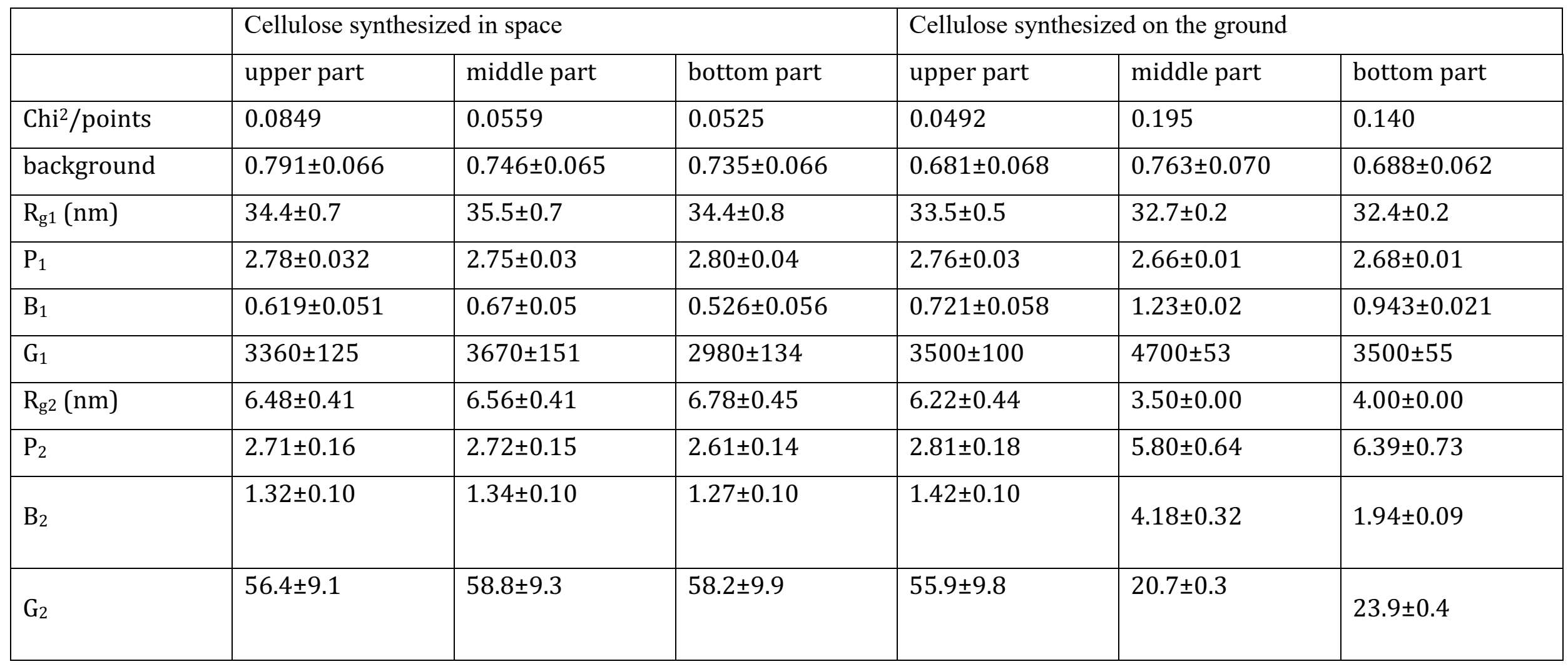

